\title{
Viviendas sobre cavernas subterráneas artificiales: una construcción social del riesgo. Caso Atizapán de Zaragoza, Estado de México
}

\author{
Houses on artificial underground caves: social construction of risk. \\ Case Atizapan of Zaragoza, State of Mexico
}

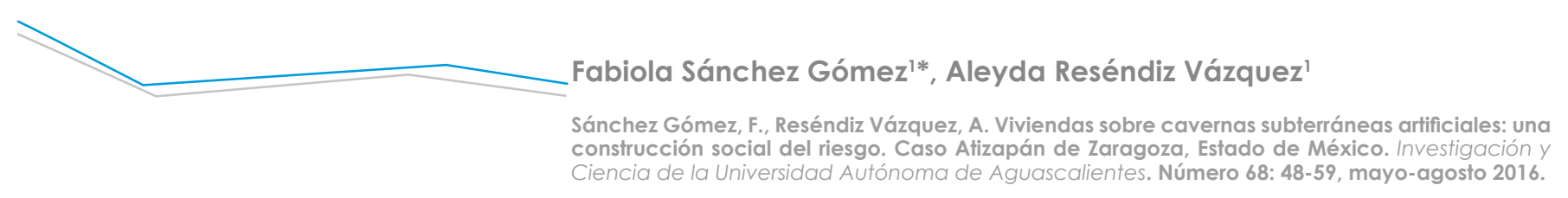

\section{RESUMEN}

La problemática de viviendas ubicadas sobre cavernas subterráneas artificiales aunada a la necesidad de vivienda en México representa un riesgo (físico, social, económico-urbano y político-jurídico), en conjunto con la vulnerabilidad de la vivienda y el crecimiento poblacional y urbano. La amenaza de subsidencia minera provoca inseguridad en la población cuando la misma se asienta en lugares en donde existen túneles mineros subterráneos. Es decir, es producto de las acciones de una población sobre la naturaleza; por tanto, un problema que se desarrolla en un proceso histórico. Las viviendas ubicadas sobre cavernas subterráneas artificiales presentan un riesgo eminente e inminente, por lo que resulta esencial la comprensión y explicación del fenómeno con base en procesos históricos e identificando los problemas urbano arquitectónicos: antes, durante y después del desastre; a partir de la interacción de dos actores principales: las inmobiliarias-municipio y la participación social.

Palabras clave: viviendas sobre cavernas subterráneas artificiales, túneles mineros subterráneos, subsidencia minera, construcción social del riesgo, amenaza, vulnerabilidad y riesgo.

Keywords: houses on artificial underground caves, underground mining tunnels, mining subsidence, social construction of risk, threat, vulnerability and risk.

Recibido: 22 de noviembre de 2014, aceptado: 13 de mayo de 2016

1 Escuela Superior de Ingeniería y Arquitectura, Instituto Politécnico Nacional, Unidad Tecamachalco.

* Autor para correspondencia: ing.arq.fabiolasanchez@hotmail.com
The problem of houses located on artificial underground caves in conjunction with the need for housing in Mexico represents a (physical, social, economic, urban and political-legal) risk; along with the vulnerability of housing and population and urban growth. The threat of mining subsidence becomes a risk when the population is settled in places on artificial underground caves. That is, risk is the product of the actions of a population on nature; therefore, a problem that develops in a historical process. The homes located on artificial underground caves have an eminent and imminent risk, so the understanding and explanation of the phenomenon is essential, based on historical processes, identifying architectural urban problems before, during and after the disaster; from the interaction of two key players: the estate-town and social participation.

\section{INTRODUCCIÓN}

La presente investigación es abordada en el ámbito arquitectónico-urbano, tomando como fenómeno las viviendas ubicadas sobre cavernas subterráneas artificiales que representan un riesgo (físico, social, económico, político, jurídico y urbano) para la población que las habita. Esto crea un riesgo social que contribuye a la creación de un desastre. La amenaza de subsidencia minera se convierte en un riesgo cuando la población se asienta en lugares con túneles mineros subterráneos. Es decir, el riesgo es producto de las acciones de una población sobre la naturaleza.

El fenómeno afrontado en la investigación tiene un campo limitado en el ámbito arquitectónico- 
urbano, es por tal razón que surge la duda: ¿Por qué se ubican viviendas sobre cavernas subterráneas artificiales? Para responder a este cuestionamiento se hace referencia a la falta de planeación en las construcciones y al descontrol de asentamientos humanos, con la finalidad de aportar información a planeaciones urbanas futuras.

Se pretende demostrar y comprobar que el riesgo de los inmuebles ubicados sobre cavernas subterráneas artificiales se construye socialmente en relación con la vulnerabilidad, a partir de la demanda de vivienda, de la política urbana, de los intereses inmobiliarios y de la percepción de riesgo de los habitantes. Para ello, se debe comprender el proceso histórico de interacción de factores involucrados para la ubicación de residencias en zonas de subsidencia minera, en conjunto con la percepción de la población que está directamente afectada, ya que la construcción social del riesgo radica en la creación y la percepción del mismo, bajo una amenaza latente.

El fenómeno se aterriza en el caso de estudio, Atizapán de Zaragoza, municipio en donde se desarrolló la actividad minera; actualmente presenta 64 zonas minadas, en donde se encuentran ubicadas casas sobre cavernas subterráneas artificiales. El municipio resulta interesante ya que es el de mayor concentración de subsidencia minera en el área metropolitana de la Ciudad de México, donde 32 son consideradas de alto riesgo, 21 de mediano y 11 de bajo. De las mismas, 22 han sido rehabilitadas, pero 32,000 personas viven en cavernas sin rehabilitar (Barrera, 2010). El Municipio de Atizapán, en el periodo comprendido desde 1930 hasta 1960 creció en expansión de asentamientos, cuando surgieron los primeros fraccionamientos. Este periodo es de suma relevancia, ya que a la mitad de la década de los cincuenta inició la explotación de yacimientos mineros, aledaños a los asentamientos de vivienda. "El periodo de auge de la extracción de yacimientos se dio después de la mitad de los cincuentas y hasta finales de los setentas" (Bringas López, 1994: 97), "lo que llevó a extraer material para la construcción de tipo arena, grava, tepetate; utilizados para el crecimiento de la Ciudad de México" (Municipio de Atizapán de Zaragoza, Protección Civil, 2009). "Al efectuarse esta actividad minera de manera descontrolada provocó la creación de cavernas subterráneas de origen artificial, dándose la formación de túneles, galerías, salones en un enjambre subterráneo, que posteriormente fueron agotadas y abandonadas" (Chávez González, 2008: 30).
En el periodo de 1960 a 1990, el municipio desarrolló la construcción en conjuntos habitacionales, unifamiliares, populares y de zona industrial. Para 1970 la explotación de yacimientos mineros descendió y la actividad minera concluyó en el territorio de Atizapán en 1982 por medio de un decreto del entonces gobernador Alfredo del Mazo que acabó de tajo con los permisos de esa actividad (Bringas López, 1994: 98).

Con este decreto se terminaron los permisos para efectuar la actividad minera, por lo que quedaron las edificaciones ubicadas sobre cavernas subterráneas artificiales y bajo la amenaza de la subsidencia minera. El crecimiento urbano en el municipio fue notable en el periodo de 1990 a 2010, debido al incremento poblacional, así como a la formación de colonias irregulares (asentamientos sin escrituras), colonias regulares y fraccionamientos. En el plano de riesgo del Plan de Desarrollo Urbano del municipio 2009-2012 (H. Ayuntamiento de Atizapán de Zaragoza, 2009) se tiene el registro de 12 zonas de minas. Ahí se identificaron zonas minadas faltantes; por lo que se relacionaron las zonas minadas en el plano de riesgo del Plan de Desarrollo Urbano del municipio de Atizapán de Zaragoza, las minas no registradas en plano de riesgo señaladas en el documento del Plan de Desarrollo Urbano Atizapán 2006-2009 (H. Ayuntamiento de Atizapán de Zaragoza, s/f) y 20092012 (H. Ayuntamiento de Atizapán de Zaragoza, 2009), las reseñas históricas y las manifestaciones registradas. Ello dio como resultado un cruce de datos para obtener la Figura 1, la cual contiene los cuatro puntos anteriores, así como las minas generales del municipio de Atizapán de Zaragoza.

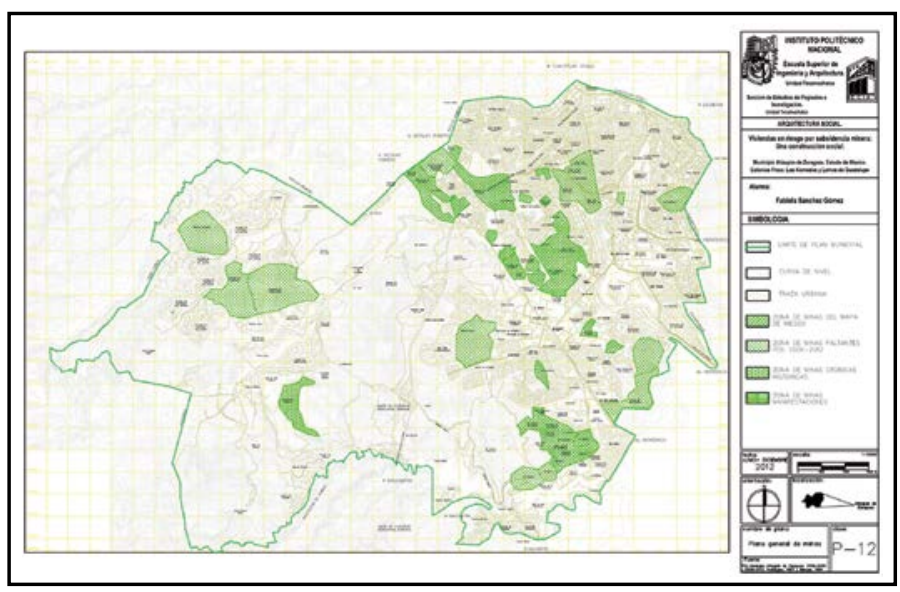

Figura 1. Plano general de minas. Elaboración propia basada en Bringas López (1994), Rodríguez Vázquez (1997) y PDU H.

Ayuntamiento de Atizapán de Zaragoza 2006-2009 (s/f) y 20092012 (H. Ayuntamiento de Atizapán de Zaragoza, 2009). 


\section{IIVESEIGGACIÓn

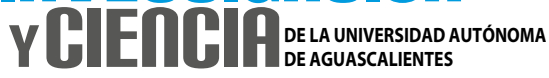

\section{MATERIALES Y MÉTODOS}

Se realizó un estudio diacrónico, en el que se presenta un análisis histórico a partir de dos casos de estudio, cuyas diferencias permitieron efectuar un análisis comparativo de cada uno de los factores intervinientes.

Para seleccionar el caso de estudio de las viviendas sobre cavernas subterráneas artificiales, se partió de tres referentes principales:

- El objeto observado: viviendas ubicadas sobre cavernas subterráneas artificiales.

- Población afectada: zonas de subsidencia minera.

- Exposición al fenómeno: determinado en mapas de riesgo y PDU de Atizapán de Zaragoza 2006 y 2012.

En cuanto a las casas afectadas sobre zonas de subsidencia minera, prevalecen dos tipologías en el municipio de Atizapán de Zaragoza: vivienda popular y media (fraccionamiento). Con lo anterior se delimitó la población y el número de residencias ubicadas sobre cavernas subterráneas artificiales en un lugar determinado. Para ello se tomaron en consideración los siguientes aspectos:

- Zonas de subsidencia minera. Donde se encuentran localizadas las colonias afectadas por el fenómeno.

- Nivel de riesgo. Permiten identificar las colonias que están sobre túneles mineros subterráneos, así como el nivel de riesgo establecido acorde a la profundidad que posee el techo de la cavidad, determinado por Protección Civil del municipio y estipulado en el PDU de Atizapán de Zaragoza 2012.

- Estatus de colonias sobre cavernas subterráneas artificiales. Se refiere a las colonias que se encuentran en zonas de subsidencia minera establecidas en el PDU de Atizapán de Zaragoza 2012, acorde a su estatus legal, ya sea regular o irregular.

- Estatus de rehabilitación-relleno de colonias sobre cavernas subterráneas artificiales. Se retomaron las colonias afectadas en zonas de subsidencia minera, establecidas en el PDU de Atizapán de Zaragoza 2012 y estipulado por Protección Civil.

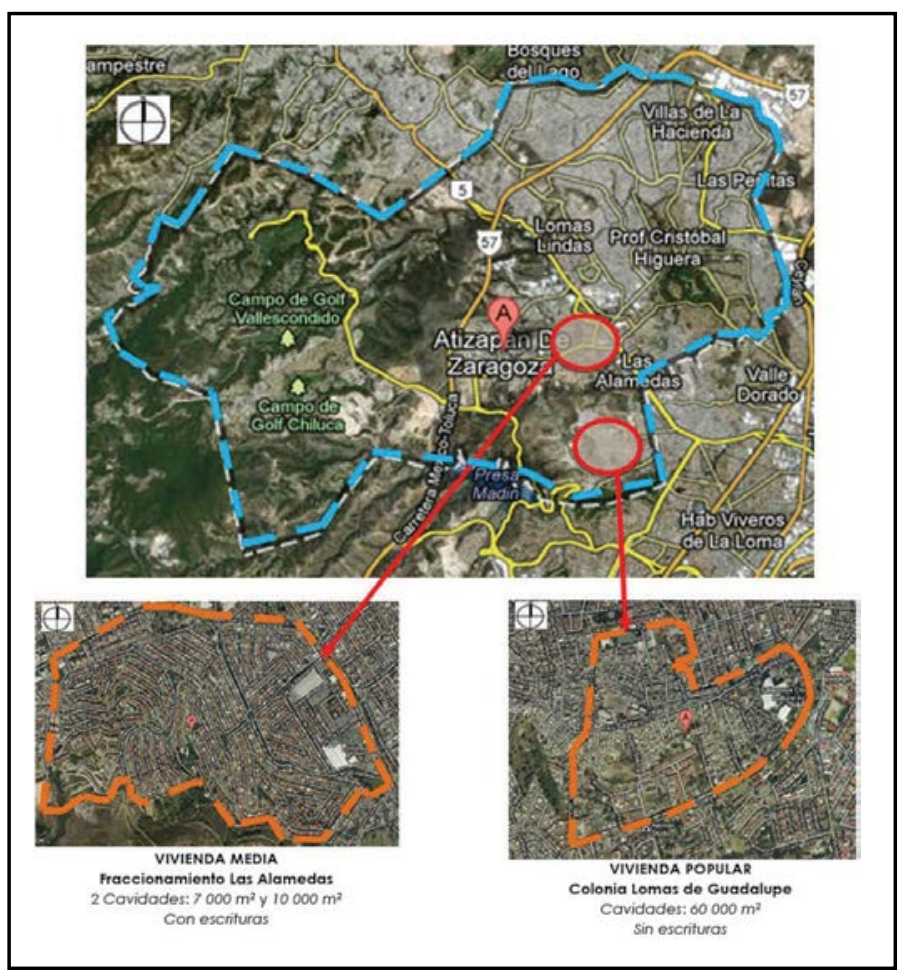

Figura 2. Selección de la muestra. Vivienda media y popular. Elaboración propia basada en Googlemaps 2012.

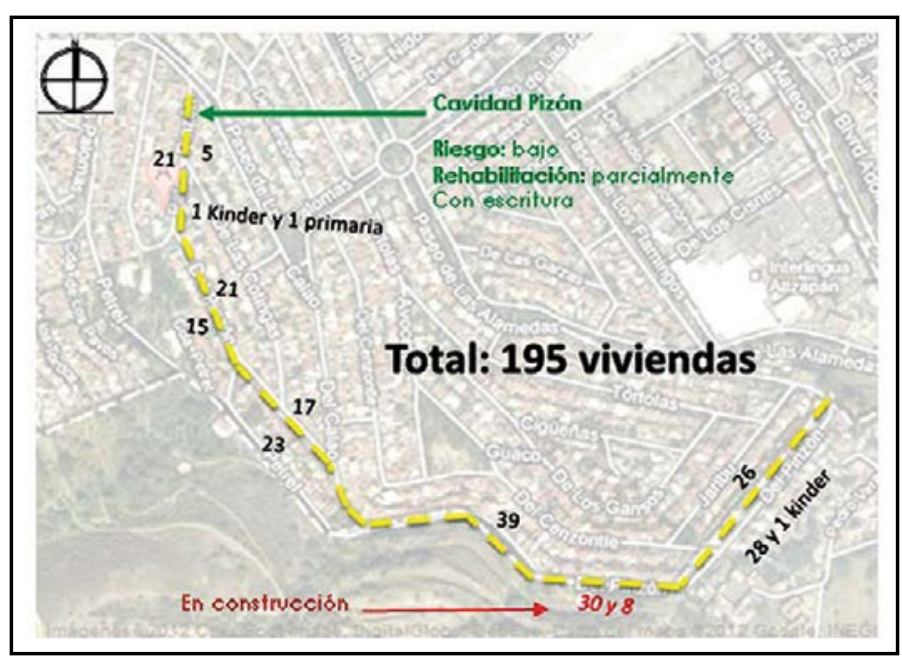

Figura 3. Fraccionamiento Alamedas, vivienda media. Ubicación de viviendas afectadas inmediatamente en la Cavidad Pizón.

Elaboración propia basada en Googlemaps 2012.

Con estos datos se seleccionó el caso de estudio de dos colonias con características muy diferentes, que permiten desarrollar una comparativa en un análisis de vulnerabilidad y percepción de riesgo.

Las colonias planteadas como caso de estudio permitieron efectuar un análisis comparativo a partir de la ubicación de casas sobre zonas de 


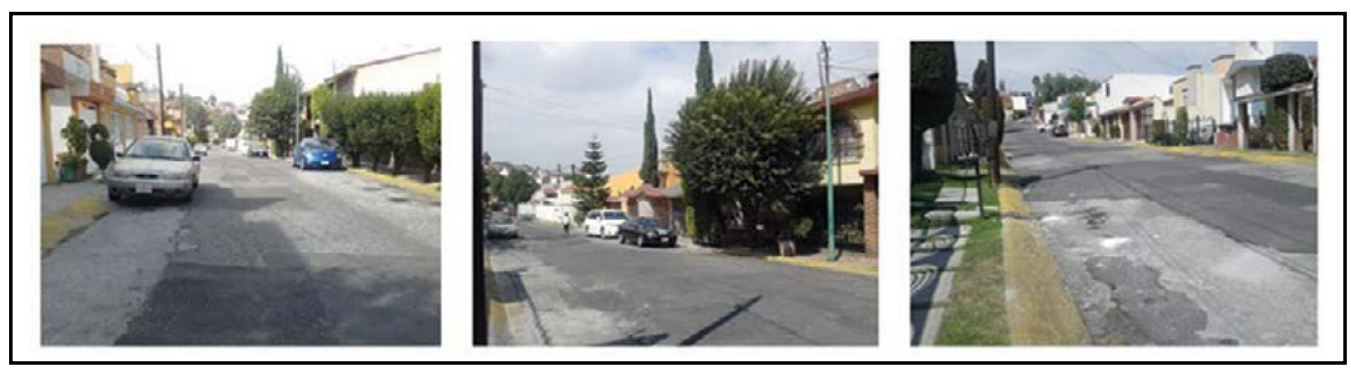

Figura 4. Fraccionamiento Alamedas. Calle por donde pasa la Cavidad Pizón. Fotografía tomada por Fabiola Sánchez Gómez.

subsidencia minera; ya que son muy diferentes en cuanto a nivel económico, tipología de vivienda, nivel de riesgo, estatus de colonia y estatus de rehabilitación-relleno.

Para efectuar la selección de la muestra se consideraron como tamaño de la población de cada caso de estudio las construcciones ubicadas sobre las cavidades antes identificadas. Para comprobar la hipótesis, fue necesario identificar las fuentes de información que son requeridas, por lo que se identificaron los actores que aportan los datos necesarios:

- Población (vivienda media: fraccionamiento y vivienda popular): el grupo inmediatamente

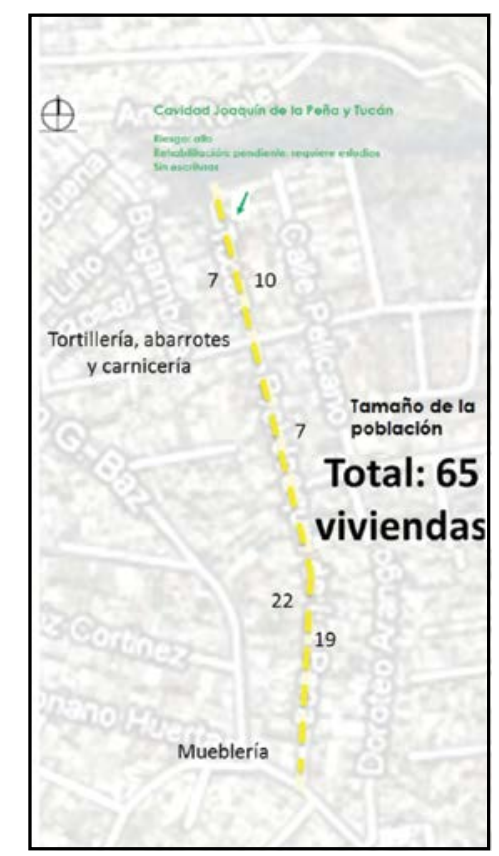

Figura 5. Colonia Lomas de Guadalupe, vivienda popular. Ubicación de viviendas afectadas inmediatamente en la Cavidad Joaquín de la Peña y Tucán. Elaboración propia basada en Googlemaps 2012. afectado, que demanda residencia debido al incremento poblacional que presenta el municipio, así como la percepción de habitar casas sobre cavernas subterráneas artificiales.

- Gobierno Municipal y Protección Civil: este grupo maneja directamente la política urbana del municipio, la cual está directamente involucrada con la edificación de residencias sobre cavernas subterráneas artificiales, así como con la protección y seguridad de quien las habita.

- Inmobiliarias: es el grupo de constructoras que edifican proyectos en el municipio, con lineamientos e interés, en particular en zonas con subsidencia minera.

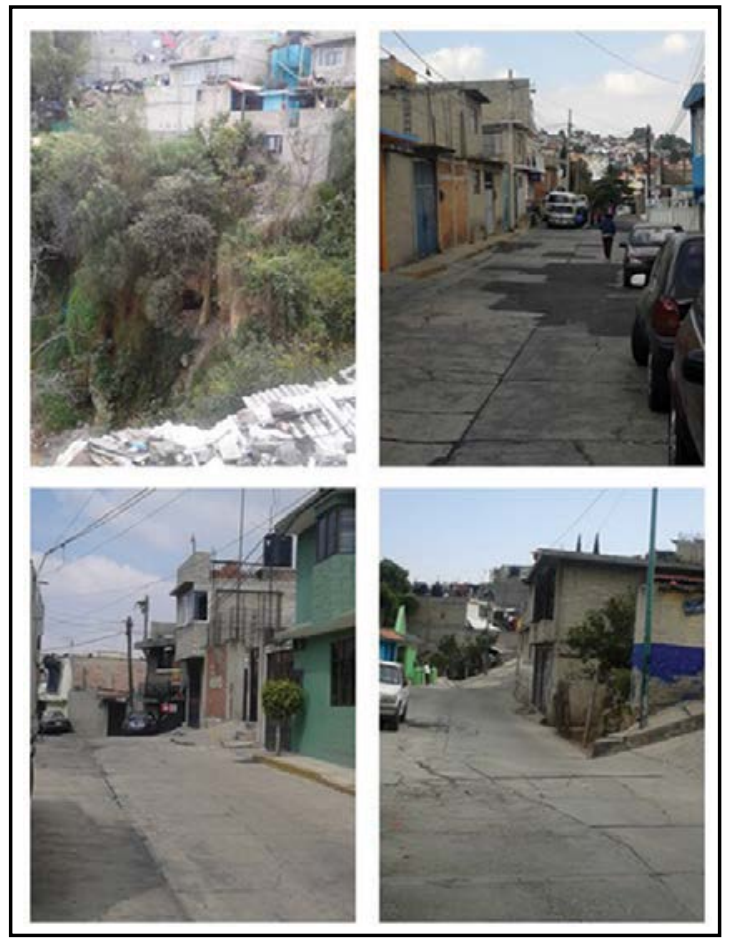

Figura 6. Colonia Lomas de Guadalupe, vivienda popular. Calle por donde pasa la Joaquín de la Peña y Tucán. Fotografía tomada por Fabiola Sánchez Gómez. 


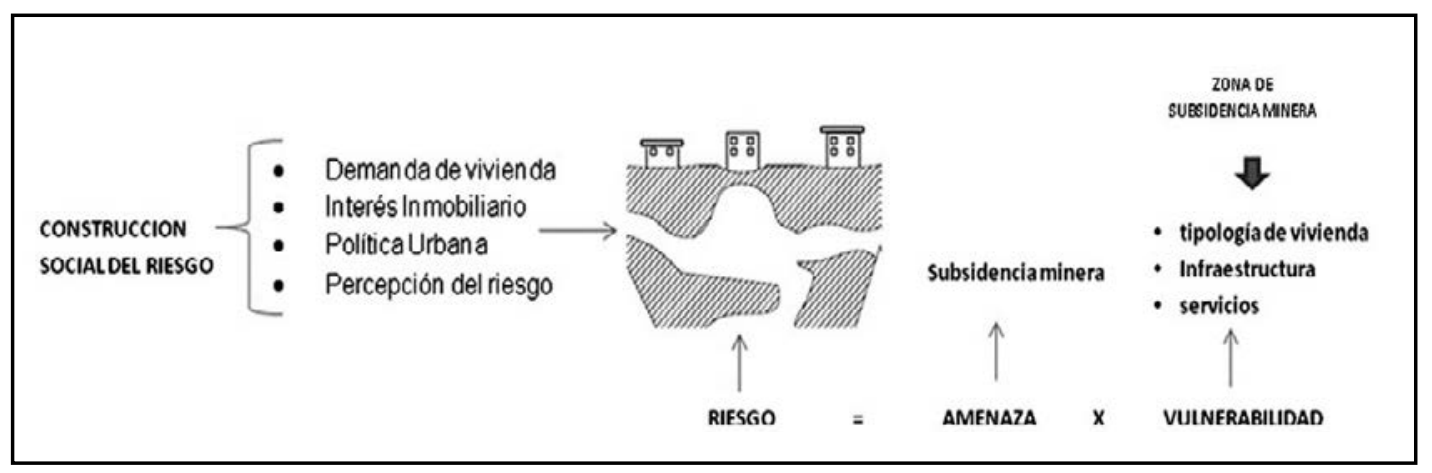

Figura 7. Construcción social del riesgo. Elaboración propia.

- Viviendas sobre cavernas subterráneas artificiales: es el grupo de edificaciones habitacionales que se encuentran ubicadas en zonas de subsidencia minera.

\section{RESULTADOS}

Se realizó un análisis del crecimiento demográfico y se relacionó con el aumento de vivienda que se ha generado históricamente en el municipio. Esto permitió explicar el aumento demográfico y crecimiento urbano en zonas con subsidencia minera, a causa de la demanda de vivienda que ha provocado la saturación de suelo para uso habitacional. Este crecimiento se ha dado, aun en zonas no aptas para uso habitacional, como es el caso de zonas con subsidencia minera. La saturación urbana ha llevado a tener actualmente una distribución de la superficie en el municipio, como se muestra en la Figura 8.

Con base en el PDU municipal 2009-2012 y en el Bando Oficial municipal 2009-2012 (H. Ayuntamiento de Atizapán de Zaragoza, 2012), se identificó el nombre de las colonias involucradas, así como la condición del estatus del predio por tipología de vivienda que se presenta en el municipio, además de aquellas colonias que no fueron entregadas y dadas de alta en el municipio como colonia ya edificada y terminada.

Se identificó la intervención de constructoras de casas en el municipio de Atizapán de Zaragoza, así como lineamientos desviados que efectúan para su edificación en zonas de subsidencia minera, por lo que se encontraron los intereses que llevan a construir de forma masiva y en zonas de cavernas subterráneas artificiales. Se realizó la consulta del análisis de mercado inmobiliario con la cobertura que posee Tasaciones Inmobiliarias (TINSA, 2012) que produce cada trimestre información detallada

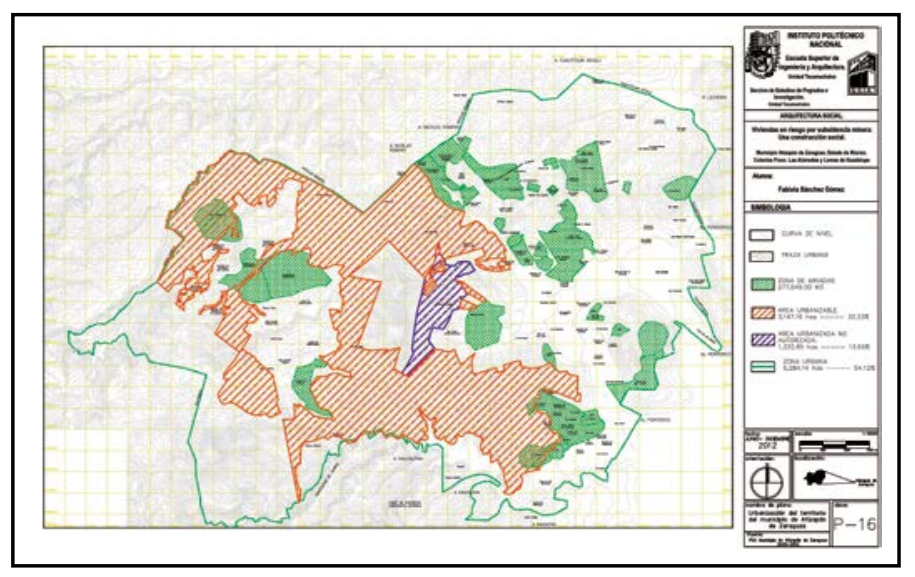

Figura 8. Urbanización del territorio del municipio de Atizapán de Zaragoza. Elaboración propia basada en PDU Municipio Atizapán de Zaragoza 2009-2012 (H. Ayuntamiento de Atizapán de Zaragoza, 2009).

sobre proyectos en venta, en donde señala que el municipio Atizapán de Zaragoza cuenta con un enfoque mercantil inmobiliario de tipo de vivienda media y residencial, a partir del año 2010 hasta 2012, en donde se considera el volumen de unidades vendidas así como el valor de venta por tipo de residencia, por lo que se tiene un aproximado de montos de operación inmobiliaria.

La producción inmobiliaria en el municipio de Atizapán de Zaragoza tuvo su auge en 2003, ya que hubo producción masiva de conjuntos habitacionales que incluso fueron construidos en zonas de subsidencia minera. La producción total de vivienda por inmobiliarias durante el periodo comprendido desde 1973 hasta 2011 fue de 57,870 construcciones, equivalente a $44.75 \%$ de la vivienda que presentaba el municipio. El PDU municipal 20092012 afirma que Atizapán de Zaragoza tiene un total de 129,300 casas. De las mismas, $69 \%$ equivale a 89,217 viviendas populares, $10 \%$ corresponde a 12,930 de tipo medio y $21 \%$ a 27,153 tipo residencial. 
Tabla 1. Producción, inversión e impuestos de inmobiliarias en el municipio Atizapán de Zaragoza 2010-2012

\begin{tabular}{|c|c|c|c|c|c|c|c|}
\hline \multirow{2}{*}{ Año } & \multirow{2}{*}{$\begin{array}{c}\text { No. de } \\
\text { proyectos }\end{array}$} & \multicolumn{2}{|c|}{ No. de viviendas } & \multicolumn{2}{|c|}{ Venta promedio } & \multirow{2}{*}{$\begin{array}{l}\text { Monto de } \\
\text { operación }\end{array}$} & \multirow{2}{*}{$\begin{array}{l}\text { Impuesto por } \\
\text { conjuntos } \\
\text { habitacionales }\end{array}$} \\
\hline & & Media & Residencial & Media & Residencial & & \\
\hline 2010 & 13 & 62 & 352 & $\$ 960,731.50$ & $\$ 1,965,132.50$ & $\$ 751,291,993.00$ & $\$ 1,512,275.52$ \\
\hline 2011 & 13 & 37 & 207 & $\$ 1,000,190.50$ & $\$ 2,045,844.50$ & $\$ 460,496,860.00$ & $\$ 890,579.52$ \\
\hline 2012 & 18 & 71 & 401 & $\$ 1,042,158.00$ & $\$ 2,131,686.50$ & $\$ 928,799,504.50$ & $\$ 1,723,652.16$ \\
\hline Total & 44 & & 1130 & & & $\$ 2,140,588,357.50$ & $\$ 4,126,507.20$ \\
\hline
\end{tabular}

Elaboración propia basada en Informe de Coyuntura Inmobiliaria, Ciudad de México 2010, 2011 y 2012 y en Código Financiero del Estado de México y municipios 2013.

En relación con los datos que proporciona el PDU municipal 2009-2012 de vivienda media y residencial más los datos obtenidos de los conjuntos urbanos desarrollados en el periodo de 1973 a 2011 con base en el Plan de Desarrollo Urbano del Estado de México, Departamento de Supervisión 2008-2012 (SDUM, 2011); se tiene que existe una variación de 17,787 residencias ante los datos presentados por el PDU municipal 2009-2012 y los datos que muestra Desarrollo Urbano del Estado de México, Departamento de Supervisión 2008-2012, de registro de viviendas media y residencial que se encuentran en el municipio que comprende a conjuntos urbanos. Para cuantificar la actividad del mercado inmobiliario en el municipio de 2010 a 2012 se presenta la Tabla 1, en donde se muestra una producción de 44 proyectos, de los cuales se realizaron 1,130 viviendas entre media y residencial. También se presenta el desglose de los tres años para los que se tienen datos de cada número de proyectos ejecutados, así como el número de casas que corresponde a cada año y tipología, la venta promedio de cada uno, el monto de operación promedio que ejercieron las inmobiliarias en relación con las edificaciones ejecutadas y el impuesto por conjuntos habitacionales que deben haber pagado por la construcción de estos.

Se analizaron los lineamientos, normas y autorizaciones que el Ayuntamiento utiliza para permitir edificar vivienda en zonas con subsidencia minera, mediante la identificación de la superficie con uso de suelo habitacional que ha sido urbanizado en zona de cavernas, así como uso de suelo manejado para crecimiento urbano.

Se efectuó una recolección de datos que permitió la identificación del uso de suelo habitacional presentado en el municipio de Atizapán de Zaragoza, lo que permitió relacionar el número de casas, superficie y porcentaje que se involucra en cada uso habitacional.

Uso de suelo habitacional + uso de suelo zona urbana $=$ Área urbanizada

$4,204.12+1,080.02=5,284.14 \mathrm{ha}$ $43.05 \%+11.04 \%=54.12 \%$

$5,284.14$ ha de área urbanizada que corresponde al $54.12 \%$ de la totalidad del territorio municipal

Tabla 2. Uso de suelo habitacional urbanizado

\begin{tabular}{|c|c|c|c|c|}
\hline Área & Uso & División & Porcentaje & \% Total \\
\hline \multirow{7}{*}{ URBANA } & \multirow{2}{*}{$\begin{array}{c}\text { Suelo } \\
\text { habitacional }\end{array}$} & Unifamiliar & $35.7 \%$ & \multirow{2}{*}{$43.05 \%$} \\
\hline & & Plurifamiliar & $7.35 \%$ & \\
\hline & \multirow{5}{*}{$\begin{array}{l}\text { Suelo zona } \\
\text { urbana }\end{array}$} & $\mathrm{CU}$ & $0.94 \%$ & \multirow{5}{*}{$11.04 \%$} \\
\hline & & IP-N & $0.50 \%$ & \\
\hline & & Equipamiento & $2.15 \%$ & \\
\hline & & Validad & $6.84 \%$ & \\
\hline & & Otros usos & $0.66 \%$ & \\
\hline \multicolumn{4}{|c|}{ Total } & $54.12 \%$ \\
\hline
\end{tabular}

Elaboración propia basada en PDU de Atizapán de Zaragoza 2009-2012 (H. Ayuntamiento de Atizapán de Zaragoza, 2009). 
Lo anterior hizo posible efectuar una comparativa del área urbanizada en Atizapán de Zaragoza en relación con el área de zonas minadas, que permite ver cómo las construcciones se han asentado en suelos de zonas de riesgo. Es por ello que se realizó una entrevista a los responsables del crecimiento urbano del municipio, así como a los encargados de dar protección y seguridad a viviendas que se encuentran sobre cavernas subterráneas artificiales. Esto dio como resultado que Desarrollo Urbano y Protección Civil del municipio Atizapán de Zaragoza carecen de sustento, congruencia y comunicación de información entre los actores municipales involucrados y en relación con el tipo de casa afectada, manejo de usos de suelo, equidad en el apoyo a la población, ubicación de zonas de subsidencia minera, actualización de datos de plano de riesgo, autorización para construir en zonas de subsidencia minera, factores que agudizan el riesgo, garantía en técnicas nuevas como seguridad y protección a la vivienda y población, así como parámetros para valorar el riesgo.

Se localizaron las casas afectadas en zonas de subsidencia minera, lo que permitió efectuar mapas para visualizar las zonas de cavernas y su rehabilitación-relleno, así como las condiciones de ocupación de vivienda que se tienen. Para realizar el análisis comparativo de la vulnerabilidad física de la construcciónsehicierongráficasquepermitenvisualizar los datos obtenidos, tanto del caso de la colonia Lomas de Guadalupe como del Fraccionamiento Las Alamedas. Se diseñó una cédula la y una cédula lb, en donde se desglosaron los dos casos de estudio con la muestra representativa para localizar las colonias de los casos de estudio que se ven afectadas por zona de subsidencia minera. Se identificó la cavidad que afecta esta zona de subsidencia minera, donde se determinó nombre de cavidad, superficie de cavidad, así como la muestra representativa de las viviendas que se involucran en cada caso de estudio. Al tener identificadas las residencias que representan la muestra al delimitar su situación, ya sea colonia regular o irregular, así como la rehabilitación-relleno en la que se encontraban, se realizó una cédula $2 a$ y una $2 b$, correspondientes a cada colonia $a$ analizar. Así se efectuó una observación directa en la muestra que se obtuvo de cada caso. Después se localizaron las viviendas en el mapa de las cédulas, se aplicaron las cédulas $3 a$ y $3 b$ de cada muestra representativa del caso de estudio de las colonias analizadas. Estas cédulas permitieron medir el nivel de vulnerabilidad física en que se encuentran las viviendas ubicadas sobre cavernas subterráneas artificiales en relación con el riesgo ya establecido, así como el contexto que lo integra y sus afectaciones al estar sobre zonas de subsidencia minera. Para ello se efectuó observación de campo y se registraron los parámetros determinados, así como los daños en grietas que permiten establecer el hundimiento presentado por medio de croquis, con observación directa y fotografía de la casa. Se estableció el análisis de la vulnerabilidad física de las viviendas que se encuentran afectadas por subsidencia minera, en un tiempo y espacio real determinado por la muestra. Para poder determinar la vulnerabilidad física de la vivienda, se consideró a Municipio de Atizapán de Zaragoza, Protección Civil (2009); Saeidi et al. (2009); Ayuntamiento de Atizapán de Zaragoza (2009) y Can et al. (2012), que permitieron determinar por medio de una valoración el nivel de vulnerabilidad física que tiene la vivienda que está ubicada sobre cavernas subterráneas artificiales. Por ello, entre mayores sean las características de la residencia y el techo de la caverna sea menor a la superficie donde está ubicada aquella el hundimiento es mayor, con grietas en la vivienda que son determinadas por la subsidencia minera.

Comoresultado hay tres niveles de vulnerabilidad en relación con los parámetros establecidos que comprenden la fragilidad física de la vivienda, como se muestra en la Tabla 3.

Para medir la vulnerabilidad que presentan las casas en las colonias de la muestra, la colonia Lomas de Guadalupe y Fraccionamiento Las Alamedas, se retomó la tabla diseñada con anterioridad y se obtuvo de manera general en porcentaje la condición vulnerable que tiene cada colonia.

\section{Vulnerabilidad física de vivienda}

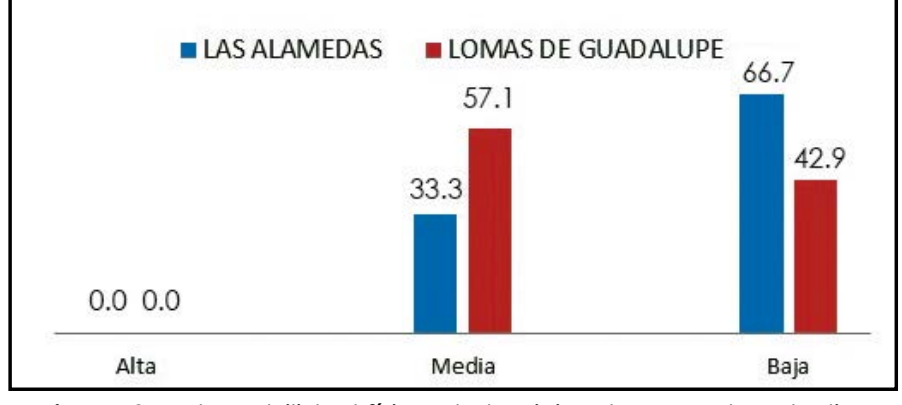

Figura 9. Vulnerabilidad física de la vivienda caso de estudio. Elaboración propia basada en resultados de parámetros para determinar la vulnerabilidad física de vivienda. 


\section{InVESEIGACIÓn

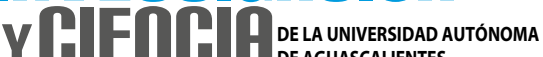

Tabla 3. Parámetros para determinar vulnerabilidad física de la vivienda

\begin{tabular}{|c|c|c|c|}
\hline \multirow{2}{*}{ Parámetros } & \multicolumn{3}{|c|}{ Vulnerabilidad } \\
\hline & Baja & Media & Alta \\
\hline \multirow{2}{*}{ Forma y simetría } & $\begin{array}{c}\text { Simple y simetría en } \\
\text { muros }\end{array}$ & $\begin{array}{l}\text { Simple y asimetría muros/ } \\
\text { irregular y simetría muros }\end{array}$ & $\begin{array}{l}\text { Irregular y asimetría } \\
\text { muros }\end{array}$ \\
\hline & 1 & 2 & 3 \\
\hline \multirow{2}{*}{ No. de plantas arq. } & 1 planta & 2 a 4 plantas & Más de 5 plantas \\
\hline & 1 & 2 & 3 \\
\hline \multirow[t]{2}{*}{ Longitud } & 7 a $10 \mathrm{~m}$ & $\begin{array}{c}\text { Mayor de } 10 \mathrm{~m} \text { y menor a } 12 \\
\mathrm{~m}\end{array}$ & Mayor de $12 \mathrm{~m}$ \\
\hline & 1 & 2 & 3 \\
\hline \multirow{2}{*}{ Material construido } & $\begin{array}{l}\text { Mampostería sin } \\
\text { refuerzo }\end{array}$ & Mampostería con refuerzo & $\begin{array}{l}\text { Mampostería y } \\
\text { concreto armado }\end{array}$ \\
\hline & 1 & 2 & 3 \\
\hline \multirow{2}{*}{$\begin{array}{l}\text { Superficie de } \\
\text { ocupación }\end{array}$} & 60 a $120 \mathrm{~m}^{2}$ & Mayor a 120 y menor a $600 \mathrm{~m}^{2}$ & Mayor a $600 \mathrm{~m}^{2}$ \\
\hline & 1 & 2 & 3 \\
\hline \multirow{2}{*}{$\begin{array}{l}\text { Superficie de } \\
\text { utilidad }\end{array}$} & 180 a $360 \mathrm{~m}^{2}$ & $\begin{array}{c}\text { Mayor a } 360 \text { y menor a } 1,200 \\
\mathrm{~m}^{2}\end{array}$ & Mayor a 1,200 m² \\
\hline & 1 & 2 & 3 \\
\hline \multirow[t]{2}{*}{ Daños en vivienda } & $\begin{array}{c}\text { Grietas estrechas/sin } \\
\text { grietas }\end{array}$ & Grietas anchas & $\begin{array}{c}\text { Grietas anchas y } \\
\text { estrechas }\end{array}$ \\
\hline & 1 & 2 & 3 \\
\hline \multirow{2}{*}{ Escrituración } & Con escrituras & En trámite & Sin escrituras \\
\hline & 1 & 2 & 3 \\
\hline Total & $8-15$ & $16-20$ & $21-24$ \\
\hline
\end{tabular}

Elaboración propia basada en H. Ayuntamiento de Atizapán de Zaragoza (2009), Municipio de Atizapán de Zaragoza, Protección Civi (2009), Saeidi et al. (2009) y Can et al. (2012).

Tabla 4. Parámetros para determinar vulnerabilidad física de la vivienda. Caso de estudio

\begin{tabular}{|c|c|c|}
\hline Características de vivienda & Las Alamedas & Lomas de Guadalupe \\
\hline \multirow{2}{*}{ Forma y simetría } & Simple y simetrías & Simple y simetrías \\
\hline & $63.2 \%$ & $65.7 \%$ \\
\hline \multirow{2}{*}{ No. de plantas arq. } & 2 a 4 plantas & 2 a 4 plantas \\
\hline & $98.2 \%$ & $74.3 \%$ \\
\hline \multirow{2}{*}{ Longitud } & $7 \mathrm{a} 10 \mathrm{~m}$ & $7 \mathrm{a} 10 \mathrm{~m}$ \\
\hline & $89.5 \%$ & $100 \%$ \\
\hline \multirow[t]{2}{*}{ Material construido } & $\begin{array}{c}\text { Mampostería y concreto } \\
\text { armado }\end{array}$ & Mampostería con refuerzo \\
\hline & $98.2 \%$ & $74.3 \%$ \\
\hline \multirow{2}{*}{ Superficie de ocupación } & 120 a $250 \mathrm{~m}^{2}$ & 60 a $120 \mathrm{~m}^{2}$ \\
\hline & $84.2 \%$ & $100 \%$ \\
\hline \multirow{2}{*}{ Superficie de utilidad } & 360 a $750 \mathrm{~m}^{2}$ & 360 a $750 \mathrm{~m}^{2}$ \\
\hline & $84.2 \%$ & $62.9 \%$ \\
\hline \multirow[t]{2}{*}{ Daño de vivienda } & $\begin{array}{c}\text { Grietas anchas (superior e } \\
\text { inferior) }\end{array}$ & $\begin{array}{c}\text { Grietas anchas (superior e } \\
\text { inferior) }\end{array}$ \\
\hline & $19.3 \%$ & $37.2 \%$ \\
\hline
\end{tabular}

Elaboración propia basada en observación de campo y Municipio de Atizapán de Zaragoza, Protección Civil (2009). 


\section{IIVESEIIGACIÓn

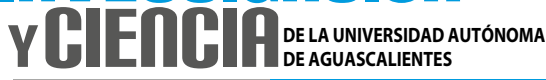

Para manejar la percepción social del riesgo se utilizaron dos indicadores principales: miedo y confianza, se formuló una encuesta que permitió medir la percepción de la población que se encuentra habitando inmuebles ubicados sobre cavernas subterráneas artificiales. Se hizo una valoración de los parámetros que determinan la percepción de riesgo que tiene una población y que da como resultado miedo o confianza, como se muestra en la siguiente tabla.

Tabla 5. Parámetros de percepción del riesgo

\begin{tabular}{|l|c|c|}
\hline \multirow{2}{*}{\multicolumn{1}{|c|}{ Parámetros de percepción }} & \multicolumn{2}{c|}{ Valoración } \\
\cline { 2 - 3 } & Sí & No \\
\hline Presencia de cavernas & 2 & 1 \\
\hline Miedo a colapso o derrumbe & 2 & 1 \\
\hline Daño a la vivienda & 1 & 2 \\
\hline Intervención de relleno & 1 & 2 \\
\hline Estudio de cavernas & 1 & 2 \\
\hline Capacitación a población & 1 & 2 \\
\hline Preparación ante el desastre & 1 & 2 \\
\hline Organización de colonia al desastre & 1 & 2 \\
\hline Reubicación necesaria & 2 & 1 \\
\hline Considerar cambiar de domicilio & 2 & 1 \\
\hline Confianza & \multicolumn{2}{|c|}{$10-15$} \\
\hline Miedo & \multicolumn{2}{|c|}{$16-20$} \\
\hline
\end{tabular}

Elaboración propia basada en Jacobson (2006) y Organización Panamericana de la Salud (OPS, 2010).
Con los datos de las encuestas y al retomar la valoración de los parámetros para la percepción del riesgo de los habitantes de las obras ubicadas sobre cavernas subterráneas artificiales, en ambos casos de estudio se presenta miedo. En las dos colonias se considera que se desarrollan en un espacio y contexto determinado, en donde la percepción del riesgo no se define por condición regular o irregular de la colonia, ni mucho menos por edad, escolaridad, ingresos y ocupación.

DISCUSIÓN

Los inmuebles ubicados sobre cavernas subterráneas artificiales son consecuencia de una demanda habitacional, ya que los resultados permitieron identificar en cada tipología de vivienda en el municipio, que las residencias populares afectadas en $33.3 \%$ se encuentran en condición irregular y $66.7 \%$ en condición regular. En lo que respecta a las viviendas tipo medio afectadas por subsidencia minera se presenta $100 \%$ en condición regular, pero $50 \%$ de las casas no fueron entregadas al municipio. Por lo que corresponde a las viviendas residenciales afectadas, $100 \%$ presenta regularidad. De lo anterior se puede concluir que parte de las viviendas ubicadas sobre cavernas subterráneas artificiales fueron asentadas por dos factores principales: demanda habitacional y venta de predios de zonas minadas.

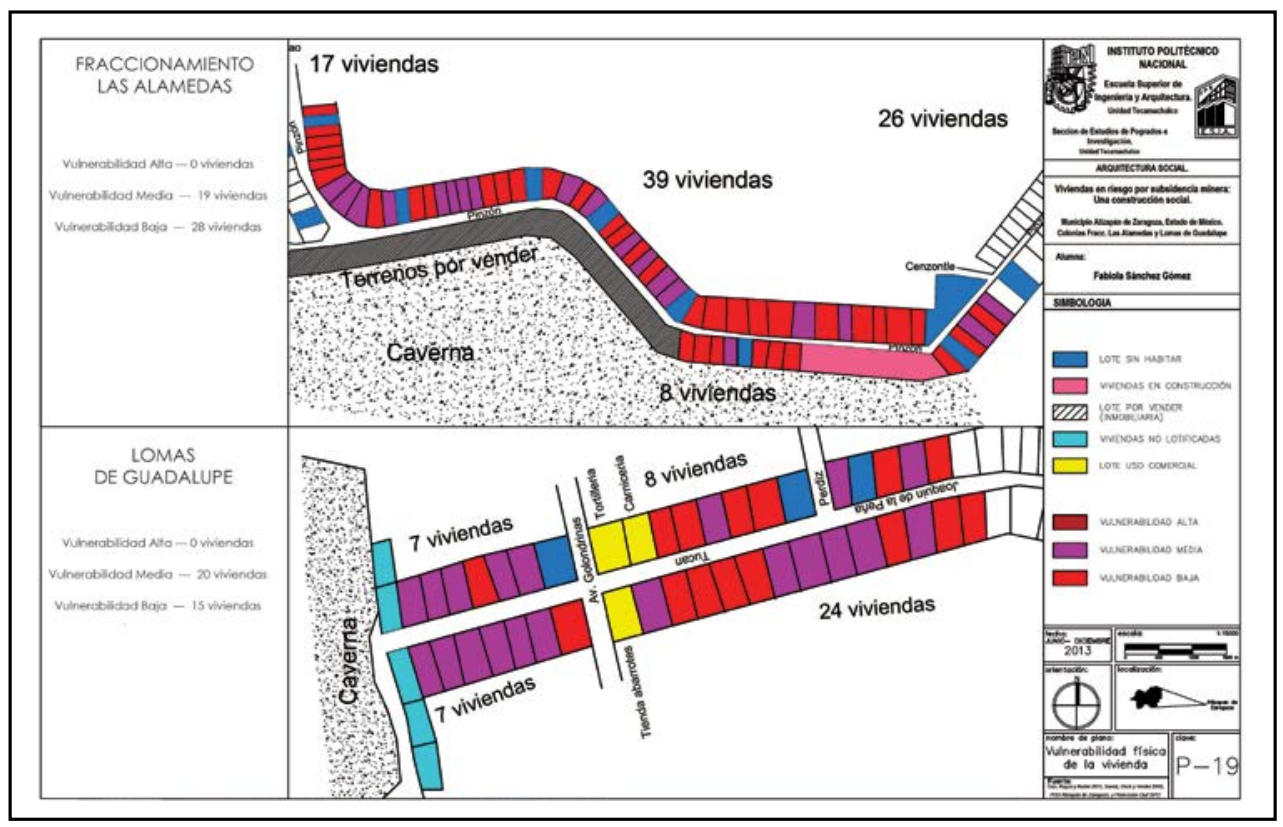

Figura 10. Vulnerabilidad física de la vivienda caso de estudio. Elaboración propia basada en resultados de parámetros para determinar la vulnerabilidad física de la vivienda. 
El primer factor se presenta a causa del incremento poblacional que se dio en el periodo comprendido desde 1960 hasta 1980 en el municipio, dejando a un lado aquella población que no tuvo la posibilidad de acceder a la casa por medio de instituciones, lo que originó la ubicación de obras en zonas de subsidencia minera, se crearon las colonias irregulares y, por tanto, la vivienda popular. El segundo factor se desarrolló después de 1982, cuando se concluyó con los permisos y explotaciones de los yacimientos mineros ubicados en el municipio de Atizapán de Zaragoza. Ello permitió después de esta fecha que los dueños de predios de zonas minadas vendieran a fraccionadores por el riesgo de que estos terrenos fueran expropiados, lo que dio como resultado la creación de las colonias regulares y, por tanto, la vivienda media, enfocada a conjuntos y fraccionamientos habitacionales. Lo anterior permitió el inicio de la producción inmobiliaria en el municipio, en donde en el año 2012 se realizaron 18 proyectos en el municipio, de los cuales 71 son de tipo medio y 401 de tipo residencial. Ahí se tiene una venta promedio en vivienda media de $\$ 1,042,158.00$, y en vivienda residencial de $\$ 2,131,686.50$, destaca que por los tres años presentados existe un incremento de valor de venta por cada tipología de 3\% cada año.

Se obtiene, por tanto, un monto de operación en el 2012, con los datos anteriores de $\$ 928,799,504.50$, de donde se debe implementar un impuesto por conjuntos habitacionales, pagado por toda persona física y jurídicas colectivas que desarrollen conjuntos urbanos, con base en el tipo de casa. Para la de tipo medio corresponden 36 salarios mínimos por vivienda y para tipo residencial 60. Todo lo anterior estipulado por el Código Financiero del Estado de México y Municipios en su última actualización el 15 de marzo de 2013. Por ello, en el año 2012 la producción inmobiliaria tuvo que dejar un ingreso anual en el municipio por el impuesto de conjuntos urbanos de un total de $\$ 1,723,652.16$, desglosado en vivienda media de $\$ 165,526.56$ y en residencial de $\$ 1,558,125.60$. Dicho ingreso debe ser registrado en el Presupuesto Municipal Definitivo de Ingresos y Egresos de Atizapán de Zaragoza 2012 (OSFEM, 2012), pero no fue así, ya que el municipio estipula que tiene un ingreso nulo del impuesto sobre conjuntos urbanos. Con lo anterior queda claro que el mercado inmobiliario en el municipio está enfocado en el tipo medio y residencial, y dicha producción habitacional tiene una variación de datos entre el PDU municipal y el DU estatal.
La inversión inmobiliaria que se ejerce en el municipio tiene un ingreso considerado en relación con el registro de casas construidas anualmente y con el impuesto de conjuntos urbanos que se deben realizar por edificarlo. Esta situación se refleja en los ingresos anuales del municipio, como se ve en el dúo de intereses inmobiliaria-municipio, por la edificación de casas en territorio municipal aun asentándose en zonas que se encuentran con subsidencia minera, ya que el municipio Atizapán de Zaragoza es el que autoriza la construcción de las viviendas en suelo con subsidencia minera; esto deja claro que busca incrementar el mercado inmobiliario enfocado en vivienda media y residencial. Por esto se deduce que la falta de regularidad en el suelo que se tiene en el territorio municipal ha provocado la ubicación de asentamientos irregulares, así como también conjuntos urbanos en zonas de subsidencia minera, por la falta de supervisión y control evidente del desarrollo urbano municipal. Ello se refleja en los casos de estudio en donde la colonia irregular carece de escrituración y de estudios y rellenos a falta de regularidad de lotes. La desocupación es mínima con respecto a la totalidad, aun con la presencia del riesgo de subsidencia minera, que principalmente se da por la falta de recursos económicos.

Por otro lado, la colonia regular, en su mayoría con escrituración, cuenta con estudios parciales, así como de relleno debido a la regularidad de los lotes. En comparación con la colonia irregular el porcentaje es elevado en vivienda desocupada, que se da no solo por la presencia del riesgo de subsidencia minera, también por la inseguridad en la colonia y la disponibilidad económica.

Se refleja la vulnerabilidad física de la casa en donde la colonia irregular cuenta con una mayoría de vulnerabilidad media de $57.10 \%$, mientras que en la colonia de fraccionamiento es una baja de 66.7\%; lo que hace notar que la colonia popular es la que cuenta con menor capacidad física a resistir el evento de algún colapso o derrumbe. Aunado a ello, que ambas colonias presenten la percepción del riesgo de miedo, ya tienen el conocimiento de la existencia y presencia de la amenaza y el riesgo. Con lo anterior se deja ver que la percepción del riesgo está determinada por la cultura de cada población en específico, mediante sus valores, costumbres, creencias y prácticas, que determinan la forma de percibir el riesgo en una población en tiempo y espacio real. 
CONCLUSIONES

La construcción social del riesgo de viviendas ubicadas sobre cavernas subterráneas artificiales se desarrolla por cinco factores principales:

- Incumplimiento y falta de regularización de leyes, normas y reglamentos de zonas de túneles mineros subterráneos.

- Interés y negocio inmobiliario, que radica en la construcción de residencias en zonas de subsidencia minera.

- Autorización de permisos de construcción por parte del municipio en túneles mineros subterráneos.

- Ineficiencia de planeación en el ordenamiento territorial ante el acelerado e incontrolado crecimiento urbano.

- La población afectada cuenta con conocimiento de las condiciones del suelo, perciben el miedo ante algún colapso o derrumbe de casa. Presentan incertidumbre ante lo que pueda suceder con el suelo y vivienda, al decidir construir y seguir habitando la zona de subsidencia minera, así como fraude al comprar terrenos en zonas de riesgo y venderlos como fraccionamientos.

Por todo lo anterior, ¿̇Qué se debe hacer para brindar protección y seguridad a viviendas ubicadas sobre cavernas subterráneas artificiales?

Es importante determinar qué se puede hacer para evitar no solo la ubicación de casas sobre cavernas subterráneas artificiales, sino también para prevenir algún desastre a las viviendas ya asentadas en zonas de subsidencia minera, aplicando políticas públicas que eviten asentamientos en zonas de subsidencia minera para evitar que se desencadenen tragedias que cobren vidas y daños materiales.

Para poder efectuar esta deconstrucción social del riesgo en construcciones ubicadas sobre cavernas subterráneas artificiales se pueden determinar aspectos que impulsen además de proporcionar protección y seguridad a los ya afectados, evitar que el fenómeno se extienda. Algunos de estos aspectos son los siguientes:

- Implementar y regularizar normas, leyes y reglamentos específicos en materia de zonas de subsidencia minera. Es decir, hacer un desglose específico en acciones de prevención, protección y seguridad en casos de construcción de vivienda, así como de la ya edificada y la población que la habita. Realizar mapas de riesgo por subsidencia minera en los que se describa concretamente el objeto-contexto, con base en una evaluación de la vulnerabilidad y la amenaza, se determinen tipo, niveles y características de las mismas. Se requeriría de una participación multidisciplinaria, por ser un fenómeno que lo amerita.

- Producción inmobiliaria que proporcione seguridad en edificaciones. Por medio de un respaldo jurídico emitir un certificado de seguridad en las viviendas edificadas, con estudios de mecánica de suelos y sistemas constructivos acorde a las características del subsuelo, así como brindar seguros de riesgo por subsidencia minera.

- Autorizaciones sujetas por parte de municipio a políticas urbanas actualizadas en materia de subsidencia minera, así como certificados de seguridad vigentes y restricción de más construcciones. Al emitir autorizaciones de construcción en zonas de subsidencia minera, se emitiría un certificado de seguridad de "no riesgo" que acreditara que el terreno está apto para edificar y no después de construido. Se deberán delimitar las zonas de subsidencia minera y destinarlas a áreas de reserva ecológica para evitar más edificación en estas.

- Impulsar y exigir una política de vivienda que permita el acceso a población no asalariada. Establecer programas que permitan brindar casas regulares a población no asalariada, así como también ubicarlas fuera de polígonos de subsidencia minera y con ello proporcionar seguridad y protección en la vivienda y la población.

- Difundir el conocimiento del riesgo por medio de una comunicación a la población afectada por subsidencia minera, enfocada en acciones de seguridad y protección. Efectuar talleres, brigadas y artículos de divulgación para la población afectada, que le permita tener el conocimiento del riesgo por subsidencia minera; así como reforzar organizaciones sociales que están en lucha por brindar casa segura y enriquecer la información del fenómeno en los portales de la red. Establecer los lazos de comunicación entre las organizaciones sociales y la población afectada para intercambiar opiniones que impulsen su seguridad y protección.

- Trabajar en una corresponsabilidad del riesgo con autoridades e instituciones que permita hacer una transferencia del riesgo por medio de acciones de seguridad y protección por cada 
actor involucrado. Proporcionar seguros de riesgo por subsidencia minera por parte de inmobiliarias, brindar certificados de seguridad de no riesgo por subsidencia minera antes de edificar por parte de autoridades y después de la entrega de la casa por parte de inmobiliarias. Participación ciudadana para tener acciones de seguridad y protección a población y vivienda en caso de desastre. Efectuar talleres, brigadas y artículos de divulgación para brindar política de prevención de riesgo a la población, en trabajo conjunto con autoridades e instituciones especializadas en el fenómeno. En cuanto a las autoridades, deberán ubicar y delimitar polígonos de zonas de subsidencia minera para convertirlos en zonas de reserva ecológica y así evitar construcciones futuras.
Con todo lo anterior se busca impulsar acciones y estrategias que permitan no solo evitar ubicación de residencias en zonas de subsidencia minera, sino también la participación de instituciones, gobiernos y población afectada en la deconstrucción social de riesgo de viviendas en zonas de subsidencia minera; así como abrir puertas a futuras investigaciones tanto en lo social, geológico y arquitectónico urbano, ya que el fenómeno de viviendas ubicadas sobre cavernas subterráneas artificiales tiene un amplio abanico de interrogantes que deben ser analizadas para dar respuestas que permitan entender el fenómeno y brindar seguridad a la población.

\section{LITERATURA CITADA}

- BARRERA, J. M. Nueva grieta es fuente de alarma en Atizapán. El Universal, 17 de septiembre de 2010.

- BRINGAS LÓPEZ, E. Atizapán de Zaragoza. Estudio Monográfico. H. Ayuntamiento Constitucional de Atizapán de Zaragoza. 1994-1996 (88-100). 1994.

- CAN., E. et al. Effects of mining subsidence on masonry buildings in Zonguldak hard coal region in Turkey. Environmental Earth Sciences, 66(8): 2503-2518, 2012.

- CHÁVEZ GONZÁLEZ, S. Atizapán: declaran alto riesgo en zona minada. La Jornada (sección Estados), 13 de agosto de 2008.

- GOBIERNO DEL ESTADO DE MÉXICO. Código Financiero del Estado de México y Municipios. Titulo Cuarto. De los ingresos de los municipios. Sección Tercera. Del impuesto sobre conjuntos urbanos (p. 262). 2013.

- H. AYUNTAMIENTO dE ATIZAPÁN DE ZARAgOzA. Bando Municipal. Ayuntamiento de Atizapán de Zaragoza, 2009-2012. Gaceta municipal, 151, 2012.

- H. Ayuntamiento de atizapÁn de zaragozA. Plan de Desarrollo Municipal de Atizapán de Zaragoza, 2006-2009. s/f

- H. Ayuntamiento de atizapÁn de zaragoza. Plan de Desarrollo Municipal de Atizapán de Zaragoza, 2009-2012. Versión Ejecutiva. 2009.

- JACOBSON, A. Percepción del riesgo y gerencia de riesgos. Ferma Education, 93, 43-49, 2006
- OSFEM (ÓRGANO SUPERIOR DE FISCALIZACIÓN DEL ESTADO DE MÉXICO). Atizapán de Zaragoza, Estado Analítico de Ingresos Presupuestales Integrado (p. 33). 2012.

- RODRÍGUEZ VÁZQUEZ, R. Tizapán. Raíces de mi pueblo. Atizapán de Zaragoza, Estado de México, México, 1997.

- SAEIDI, A. et al. Development of Building vulnerability functions in subsidence regions from empirical methods. Engineering Structures, 31 (10): 2275-2286, 2009

- TINSA (TASACIONES INMOBILIARIAS). Ventas y stock de viviendas nuevas. Mercado habitacional de la zona metropolitana de la ciudad de México. INCOIN Informe de Coyuntura Inmobiliaria, 3er trimestre 2012.

\section{De páginas electrónicas}

- MUNICIPIO DE ATIZAPÁN DE ZARAGOZA, ESTADO DE MÉXICO. DIRECCIÓN GENERAL DE PROTECCIÓN CIVIL Y BOMBEROS. Riesgos Geológicos. Protección Civil. 2009. Recuperado el 22 de abril de 2011, de http://www.facebook.com/ PCEYBAtizapan/? fref=ts

- OPS (ORGANIZACIÓN PANAMERICANA DE LA SALUD). Módulo 2: Percepción de riesgos. Curso de autoaprendizaje en comunicación de riesgos. 2010. Recuperado el 29 de marzo de 2012, de http://www.bvsde.paho.org/cursocr/e/modulo2.php

- SDUM (SECRETARÍA DE DESARROLLO URBANO Y METROPOLITANO). Sitios de interés en Autorizaciones de Conjuntos Urbanos 2010-2012. Recuperado el 29 de marzo de 2012, de http://sedur.edomex.gob.mx.autorizaciones 